\title{
Functional assessment of a new extended depth-of-focus intraocular lens
}

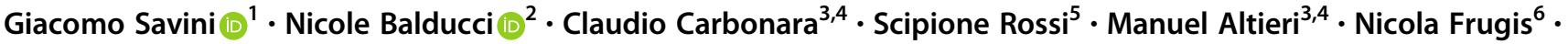 \\ Emilia Zappulla ${ }^{5} \cdot$ Roberto Bellucci $^{7} \cdot$ Giovanni Alessio $^{6}$
}

Received: 2 April 2018 / Revised: 6 August 2018 / Accepted: 23 August 2018 / Published online: 28 September 2018

(c) The Royal College of Ophthalmologists 2018

\begin{abstract}
Background/objective This study aimed to investigate the visual performance of a new extended depth-of-focus intraocular lens (EDOF-IOL).

Subjects/methods In this multicenter, prospective, observational study, we enrolled 97 patients who underwent cataract surgery or refractive lens exchange with implantation of the Mini Well EDOF-IOL (SIFI, Italy). Patients underwent postoperatively the following examinations between 4 and 8 weeks after surgery: corrected distance visual acuity (CDVA), reading speed with Radner's chart, distance-corrected near visual acuity (DCNVA), defocus curve, contrast sensitivity, and haloes quantitative assessment.

Results In the whole sample, the mean monocular CDVA and DCNVA were, respectively, $0.02 \pm 0.07 \log$ AR and $0.38 \pm$ $0.15 \operatorname{logRAD}$ (logarithm of the reading acuity determination). In the 67 bilaterally implanted patients, binocular CDVA and DCNVA were better $(0.00 \pm 0.05 \log$ MAR and $0.26 \pm 0.13 \operatorname{logRAD})$ than the corresponding monocular values $(p=0.02$ and $p=0.0002$, respectively). Ninety-two percent of patients bilaterally implanted reached a binocular reading speed $>80$ words per minute at a $0.5 \operatorname{logRAD}$ print size (corresponding to the common book print size). The defocus curves showed that the EDOF-IOL provided increased depth of focus through 2.0 D of defocus, with the best performance at 1.0 and $1.5 \mathrm{D}$. Contrast sensitivity was within normal limits at all spatial frequencies. The mean visual disturbance index was $0.08 \pm 0.12$, suggesting low night visual disturbances.

Conclusions The new EDOF-IOL provided good visual acuity for distance, intermediate, and near vision, with no loss of contrast sensitivity and low risk of night visual disturbances.
\end{abstract}

\section{Introduction}

Different multifocal intraocular lenses (MF-IOLs) can be used to correct presbyopia at the time of cataract removal or refractive lens exchange. Traditionally, most MF-IOLs acted as bifocal IOLs, that is, they formed two primary

Giovanni Alessio

giacomo.savini@alice.it

IRCCS - Fondazione Bietti, Rome, Italy

2 Studio Oculistico d'Azeglio, Bologna, Italy

3 Clinica Villa Valeria, Rome, Italy

4 Clinica Nuova Itor, Rome, Italy

5 San Carlo di Nancy Hospital, Rome, Italy

6 Unità Operativa Oftalmologia Universitaria, Dipartimento di Scienze Mediche di Base, Neuroscienze ed Organi di Senso, University of Bari "Aldo Moro", Bari, Italy

7 Ophthalmic Unit, University Hospital, Verona, Italy focal points providing functional distance and near vision. The near add usually ranged between +3.0 diopters (D) and $+4.0 \mathrm{D}$ at the IOL plane and in the average eye enabled near focal distance between 36 and $48 \mathrm{~cm}$ [1]. However, these IOLs were found to provide poor visual function at intermediate distances, especially with the highest degrees of near add [2-4]. For this reason, manufacturers started developing MF-IOLs with lower near add. For example, the Restor IOL (Alcon Labs, Fort Worth, TX, USA) was first marketed with $+4.0 \mathrm{D}$ near add, then with $+3.0 \mathrm{D}$ near add and finally with $+2.5 \mathrm{D}$ near add. MF-IOLs with low near add (e.g., +2.5 or $+2.75 \mathrm{D}$ ) improve vision at intermediate 
distances [5-7], which are important in the era of computers and smartphones, but reduce the ability to read at closer distances, which is very important for common tasks such as newspaper reading [8]. Different MF-IOL designs have been developed to provide a continuous range of vision and thus minimize the typical through-focus V pattern of MFIOLs with two foci, such as trifocal IOLs and extended depth-of-focus IOLs (EDOF-IOLs) [9-11]. Within the latter category, one of the most interesting and innovative solutions is provided by the Mini Well IOL (SIFI, Catania, Italy), which has been developed to extend the depth of focus by inducing spherical aberrations. Different studies at the optical bench have shown that it increases the depth of focus [12-14]. In the first published clinical study, we recently reported that it shows excellent visual outcomes at intermediate distances and induces less haloes than a distance-dominant diffractive MF-IOL [15]. The present study was designed to investigate the visual performance in a larger group of patients implanted with this IOL.

\section{Materials and methods}

\section{Patients}

In this prospective observational multicenter study, pseudophakic patients who had received the new Mini Well EDOF-IOL at least in one eye were enrolled. The study methods adhered to the tenets of the Declaration of Helsinki for the use of human participants in biomedical research and were approved by the local ethical committees. Informed consent was obtained from each patient. The study protocol was registered in clinicaltrials.gov as NTC02740010.

Exclusion criteria were any corneal disease (e.g., keratoconus, epithelial basal membrane dystrophy, scars, etc.), or other ocular comorbidity that could influence postoperative evaluations, previous eye surgery, any intraoperative complication, reduced zonular/capsular stability (e.g., pseudoexfoliation), keratometric astigmatism $>0.75 \mathrm{D}$, and poor ability to perform the tasks included in the postoperative tests necessary for the present study.

The following examinations were performed before surgery: slit lamp evaluation, Goldmann applanation tonometry, dilated fundus evaluation, axial length measurement by optical biometry or immersion ultrasound biometry, manifest refraction, monocular and binocular corrected distance visual acuity (CDVA), and uncorrected distance visual acuity (UDVA) assessed using the Early Treatment of Diabetic Retinopathy Study (ETDRS) chart at $4 \mathrm{~m}$. Moreover, corneal topography and scotopic and photopic pupillometry were performed using a rotating Scheimpflug camera combined with a Placido disk corneal topographer (Sirius, Costruzione Strumenti Oftalmici, Florence, Italy).

\section{Intraocular lens}

The Mini Well is a progressive EDOF-IOL with an optical design based on wavefront engineering. The technical innovation consists of the implementation of positive and negative spherical aberrations in the central part of the optic, in order to increase the depth of focus and generate a continuum of foci [16]. It is a single-piece, aspheric biconvex hydrophilic-hydrophobic copolymer IOL with three different optical zones. The inner zone induces a positive spherical aberration, the intermediate zone induces a negative spherical aberration, and the outer zone has a monofocal aspherical design. The transitions between the three optical zones are smooth and present a gradual power shift (the so-called "active transition zones") in order to support a progressive vision. The optic diameter is $6 \mathrm{~mm}$, the overall diameter is $10.75 \mathrm{~mm}$, and the vault is $5^{\circ}$.

\section{Surgical technique}

Phacoemulsification and IOL implantation were performed by expert surgeons through a temporal near-to-clear corneal sutureless $2.2 \mathrm{~mm}$ incision under topical and intracameral anesthesia (1\% preservative-free lidocaine). The IOL was implanted in the capsular bag and centered on the first Purkinje reflex.

\section{Postoperative examinations}

Between 4 and 8 weeks after surgery, all patients underwent the following examinations (patients implanted in both eyes were assessed both in monocular and in binocular vision).

The manifest refraction was determined clinically. Monocular and binocular UDVA and CDVA were measured using the ETDRS chart at $4.0 \mathrm{~m}$. Near vision assessment was performed with the Radner Reading Chart (Italian validated version) under bright light conditions $\left(100 \mathrm{~cd} / \mathrm{m}^{2}\right)$ [17]. Monocular and binocular distancecorrected near visual acuity (DCNVA) at $40 \mathrm{~cm}$ was expressed in $\log \mathrm{RAD}$ (logarithm of the reading acuity determination, the reading equivalent of $\log M A R)$. The reading speed (words per minute (WPM)) was calculated using the formula reading speed $=$ number of words in the sentence/time in seconds needed to read the sentence. The critical print size (CPS) is the smallest print size the patients were able to read with the maximum reading speed [17-19].

The defocus curves (visual acuity (VA) over imposed defocus) were recorded under photopic conditions by adding negative lenses in half-diopter steps up to $-4 \mathrm{D}$ and positive lenses up to $+1 \mathrm{D}$ to the distance-corrected manifest refraction $[9,20]$. In this curve, the intermediate vision corresponds to -1.5 and $-1 \mathrm{D}$ defocus. The DCNVA corresponds to $-2.5 \mathrm{D}$ defocus. 
Table 1 Postoperative data of the 67 patients with bilateral EDOF-IOLs and comparison of the clinical performance obtained from the right eye (RE) to that obtained binocularly

\begin{tabular}{llll}
\hline & Monocular $(\mathrm{RE})(n=67)$ & Binocular $(n=67)$ & $p$ value* \\
\hline Uncorrected distance visual acuity (logMAR) & $0.09 \pm 0.11$ & $0.03 \pm 0.07$ & 0.0021 \\
CDVA (logMAR) & $0.02 \pm 0.07$ & $0.00 \pm 0.05$ & 0.02 \\
DCNVA (logRAD) & $0.39 \pm 0.16$ & $0.26 \pm 0.13$ & 0.0002 \\
CPS (logRAD) & $0.57 \pm 0.15$ & $0.48 \pm 0.15$ & 0.0053 \\
\hline
\end{tabular}

$\log M A R$ logarithm of the minimum angle of resolution, $C D V A$ corrected distance visual acuity, $D C N V A$ distance-corrected near visual acuity, $\log R A D$ logarithm of the reading acuity determination, $C P S$ critical print size

*Mann-Whitney test
Monocular distance-corrected contrast sensitivity (CS) was measured using a computer screen enabling the presentation of sine-wave gratings at different spatial frequencies $(1.5,3,6,12$, and 18 cycles per degree (cpd)) [21]. This computer has a background illumination calibrated at $85 \mathrm{~cd} / \mathrm{m}^{2}$ and thus provides independence from room illumination. It also gives a normative range at all spatial frequencies, based on the same normative values used by other instruments such as the CSV-1000E (VectorVision, Greenville, OH, USA) [22]. Absolute values of $\log _{10} \mathrm{CS}$ were obtained for each combination of eye and spatial frequency, and means and standard deviations were calculated.

The presence of visual disturbances under low illumination was tested monocularly with specific software (Halo v1.0 software, Laboratory of Vision Sciences and Applications, University of Granada, Spain) [23-25]. In this test, the observer task is to discriminate, on a monitor, the peripheral bright stimuli around a small central highluminance stimulus over a dark background. The central bright stimulus causes a certain amount of intraocular scattering and retinal reflection in the patient's eye, depending on the state of the ocular media of the subject. This effect is expected to increase with any MF-IOL or EDOF-IOL provoking visual disturbances, such as glare and visual halos perceived by the observer. This software is able to quantify visual disturbances by means of the disturbance index, that is, the ratio between non-detected stimuli and all the peripheral stimuli presented to the observer as a number between 0 and 1 . The higher is the index, the lower the discrimination capacity. Therefore, a high index means that it is more difficult for the subject to detect the peripheral stimuli near the central stimulus, which is in turn indicative of a greater influence of halos or night-vision disturbances. The test was performed at a distance of $2 \mathrm{~m}$ with a $1024 \times 768$ pixel resolution on the monitor and the same parameters previously reported (the size of the stimuli was 20 pixels for the radius of the main stimulus and 2 pixels for the radius of the peripheral stimuli). The monitor showed a total of 36 peripheral stimuli around the central one, distributed along 12 semiaxes (three stimuli per semiaxis). The maximum radius of each semiaxis was
50 pixels (the most distant stimulus being 50 pixels from the center of the main stimulus) [24].

In addition, patients were asked if they were suffering from visual disturbances at night, including glare, haloes, starbursts, hazy vision, monocular polyopia, simultaneous vision, and defocus. For this purpose, we relied on the images and the scale described by McAlinden et al. [26]

Statistical analysis was performed in order to compare monocular and binocular VA and reading parameters. Since the data did not pass normality tests, the Mann-Whitney test for nonparametric data was used. Moreover, multiple linear regression was performed to look for any correlation between biometric parameters and near VA. The statistical analysis was performed using the SAS software (V.9.2, SAS Institute Inc.). A $p$ value $<0.05$ was considered to be statistically significant.

\section{Results}

One hundred patients were recruited. Three patients were excluded from analysis due to missing follow-up data. Therefore the analyzed data set included 97 patients (164 eyes). Their mean age was $68.57 \pm 0.98$ years (range: 45-91; males:females $=47: 50$ ). The EDOF-IOL was implanted in both eyes in 67 patients $(69.1 \%)$ and in one eye of the remaining 30 patients (30.9\%). The power of the implanted EDOF-IOL ranged from +10 to $+29 \mathrm{D}$ (mean: $21.12 \pm 2.82 \mathrm{D})$. Preoperatively, the mean corneal power was $43.62 \pm 1.57 \mathrm{D}$, the mean corneal asphericity ( $Q$ value at $8 \mathrm{~mm}$ ) was $-0.23 \pm 0.16$, the mean axial length was $23.47 \pm 1.09 \mathrm{~mm}$, the mean photopic pupil diameter was $3.03 \pm 0.66 \mathrm{~mm}$, the mean scotopic pupil diameter was 4.15 $\pm 0.99 \mathrm{~mm}$, and the mean pupil decentration was $0.20 \pm$ $0.23 \mathrm{~mm}$.

Postoperatively, in the whole sample the mean monocular UDVA and CDVA were, respectively, $0.11 \pm 0.12$ and $0.02 \pm 0.07 \log$ MAR; the mean monocular DCNVA was $0.38 \pm 0.15 \operatorname{logRAD}$ and the mean CPS was $0.55 \pm 0.16$ $\log \mathrm{RAD}$. Table 1 reports the postoperative data of patients who received the EDOF-IOL in both eyes and compares the clinical performance obtained from just one eye (the right 


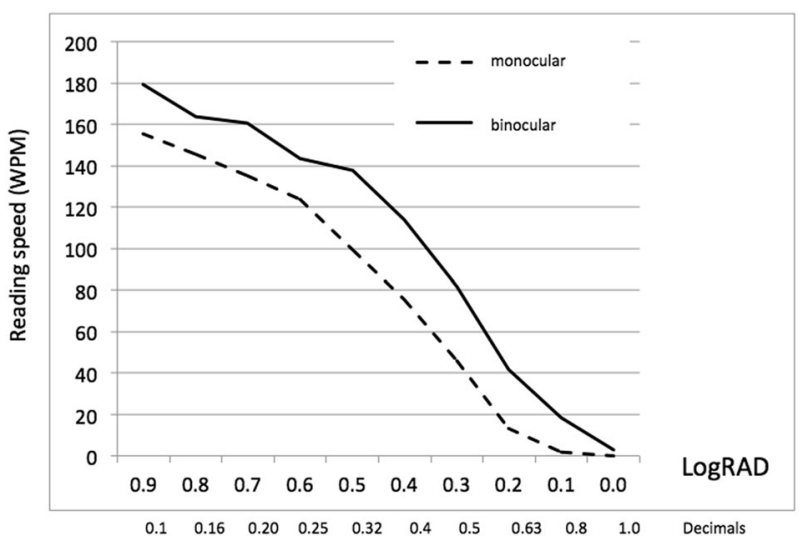

Fig. 1 Mean values of monocular and binocular reading speed obtained at different print sizes with the Mini Well EDOF-IOL. WPM words per minute, $\operatorname{logRAD} \operatorname{logarithm}$ of reading acuity determination

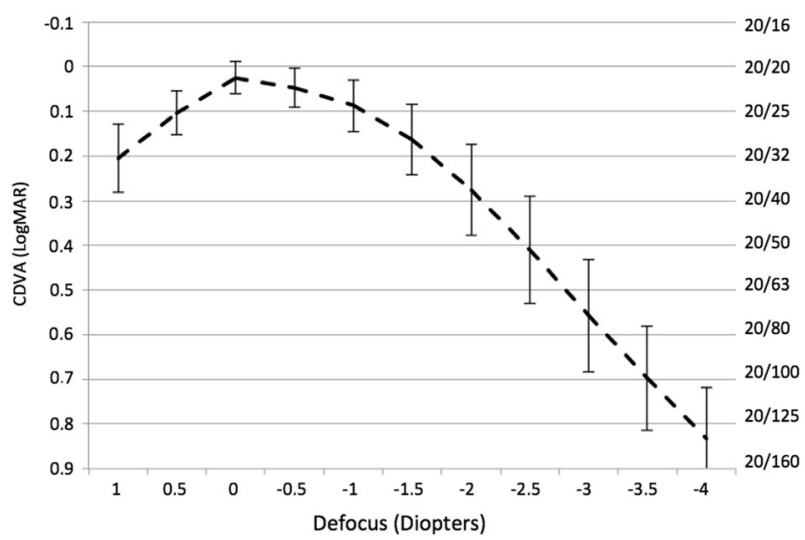

Fig. 2 Monocular defocus curve in patients implanted with the Mini Well EDOF-IOL. The vertical bars represent the standard deviation at defocus. CDVA corrected distance visual acuity

eye) to that obtained binocularly. Results were statistically better when VA and reading parameters were evaluated binocularly than when they were evaluated monocularly.

Figure 1 shows the mean values of the monocular $(n=164)$ and binocular $(n=67)$ reading speed for sentences in different print sizes. Considering $80 \mathrm{WPM}$ as the minimum reading speed for fluent reading [27], 92\% of the patients with bilateral EDOF-IOLs reached a binocular reading speed $>80 \mathrm{WPM}$ at a $0.5 \operatorname{logRAD}$ print size (which corresponds to the common book print size).

The monocular defocus curve for all 164 eyes shown in Fig. 2 reveals gradually decreasing VA for increasing negative defocus levels, with a continuous performance and no VA gaps for the intermediate range. A mean VA of 20/40 or better was obtained through $2 \mathrm{D}$ of defocus: a mean VA of 0.8 decimals $(\log \mathrm{MAR}=0.09 \pm 0.12)$, 0.7 decimals $(\log \mathrm{MAR}=0.17 \pm 0.15)$, and 0.53 decimals $(\log \mathrm{MAR}=0.28 \pm 0.20)$ was recorded at $-1.0,-1.5$, and $-2.0 \mathrm{D}$ of defocus, respectively. Biometric parameters, such as corneal power, anterior chamber depth, and axial

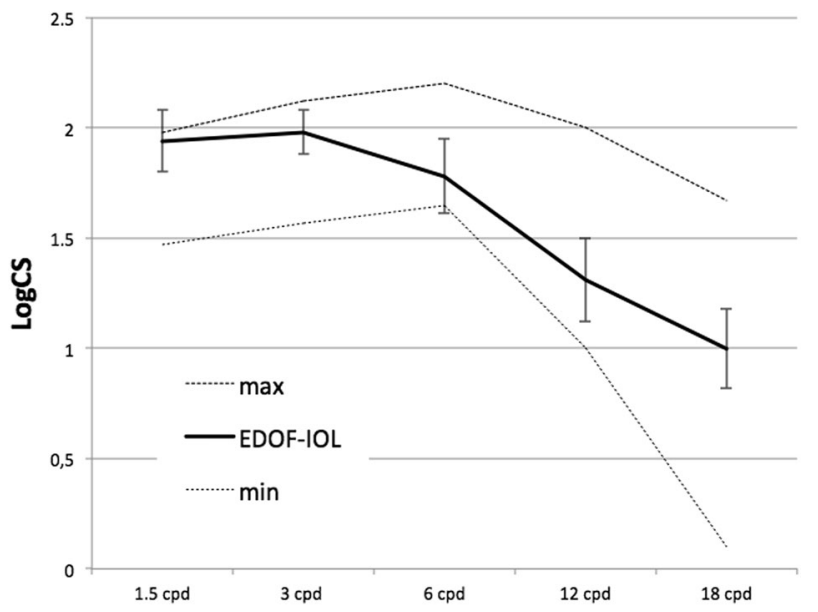

Fig. 3 Contrast sensitivity of the Mini Well EDOF-IOL. The dotted lines represent the minimum $(\min )$ and maximum $(\max )$ limits of the normative database. The vertical bars represent the standard deviation at each frequency

length, did not influence the near VA, as no statistically significant correlations were found between the abovementioned parameters and VA with a defocus of -1.0 , $-1.5,-2.0$, and $-2.5 \mathrm{D}$.

The mean values of monocular CS (expressed as $\log _{10}$ CS) were $1.93 \pm 0.26$ at $1.5 \mathrm{cpd}, 1.98 \pm 0.21$ at $3 \mathrm{cpd}, 1.82$ \pm 0.29 at $6 \mathrm{cpd}, 1.28 \pm 0.40$ at $12 \mathrm{cpd}$, and $0.98 \pm 0.38$ at $18 \mathrm{cpd}$. As shown in Fig. 3, these values were within normal limits according to the normative database of the CS test.

As regards the objective halo assessment, the mean monocular disturbance index (quadratic) was $0.08 \pm 0.12$ (range: $0.00-0.65$ ). Mild bilateral haloes (grade 1) and glare (grade 1) at night were reported, respectively, by four $(4.1 \%)$ and seven $(7.2 \%)$ patients. These subjects, however, refused the opportunity to exchange the IOL because the disturbance was acceptable in their opinion.

\section{Discussion}

This study was designed to clinically confirm the early promising results of Mini Well, a new EDOF-IOL [12-16]. A preliminary study on a smaller sample of patients $(n=20)$ found that it offers better intermediate vision and induces less haloes with respect to a distance-dominant diffractive MF-IOL. These advantages are not counterbalanced by a loss of CDVA, near vision, and CS [15]. The data of the present study, obtained from 164 eyes, confirm that this EDOF-IOL can correct presbyopia without compromising the distance VA and with a minimal risk of visual disturbances at night. The latter issue is the most important finding, as the occurrence of haloes and glare is one of the most annoying complications of MF-IOLs and a leading cause for explantation [28]. 
The distance VA was excellent, as revealed by the mean CDVA value, which was close to that recently reported [15]. Not surprisingly, this parameter was slightly better $(0.0 \pm 0.05 \log$ MAR $)$ when measured binocularly. The monocular and binocular CDVA after implantation of the Mini Well EDOF-IOL is similar to that measured after implantation of other MF-IOLs [6, 7, 10, 18, 29, 30]. The mean UDVA was good, but this parameter is influenced by the prediction error in IOL power calculation and does not depend on the optical quality of the IOL. Hence its value in this context is limited.

The analysis of VA at closer distances, clearly illustrated by the defocus curve, reveals that the Mini Well EDOF-IOL provides its best outcomes at intermediate distances, since VA is maintained over the level of 20/40 (0.28 LogMAR) up to $2 \mathrm{D}$ of defocus. The typical gap that occurs in the defocus curve of traditional "bifocal" MF-IOLs, as a consequence of the reduced VA at intermediate distances $[2-4,20]$, is not observed in Fig. 2. This finding is in good agreement with our preliminary study [15], and demonstrates that the Mini Well EDOF-IOL provides patients with true EDOF and represents one of the best choices for patients looking for good VA for distances between 40 and $100 \mathrm{~cm}$. The enhanced performance at intermediate distances is ascribable to the optic of the IOL, which is not designed to provide different foci by diffraction or refraction, but rather to generate a continuum of foci by the implementation of spherical aberration and the presence of optically active transitional zones. The defocus curve that most closely resembles that of the Mini Well EDOF-IOL is that of the Crystalens AO IOL, a pseudo-accommodative IOL with totally different mechanism of action [20]. Similarly to the Mini Well EDOF-IOL, the Crystalens AO IOL produces no VA gaps at intermediate distances; it achieves similar DCNVA with $-1.0 \mathrm{D}$ defocus (0.1 logMAR), but lower DCNVA with -2.0 D defocus (0.4 logMAR) [20]. At a closer reading distance, the clinical performance of the Mini Well can still be considered good, given that at $40 \mathrm{~cm}$ more than $90 \%$ of bilaterally implanted patients were able to fluently read a text corresponding to the common newspaper print size.

The clinical results of this study are in good agreement with the in vitro measurements recently reported by Domínguez-Vicent et al. [12, 13] and Bellucci et al. [14], who investigated the optical properties of the Mini Well. In fact, the good performance achieved in our population at near and intermediate distance can be explained by the broad intermediate-near area and large defocus tolerance observed with through-focus modulation transfer function curves [12-14]. On the other hand, the lack of visual disturbances at night, which has been already reported in our preliminary study [15], can be explained both by the absence of diffraction rings in the IOL optics and by the outstanding image quality observed with the modulation transfer function curve at $0.0 \mathrm{D}$ for large pupil diameter (4.5 mm) [12, 13].

As far as the quality of vision is concerned, the patients implanted with the Mini Well EDOF-IOL did not suffer from a loss of $\mathrm{CS}$, as the mean values were within the normative range for all tested frequencies. The values found in this study at each spatial frequency were equal to or better than those previously reported for other MF-IOLs when tested for photopic CS [10, 31].

Visual disturbances and photic phenomena were not complained by the great majority of patients. Less than $10 \%$ of patients complained of subjective disturbances at night. This percentage confirm our previous findings [15] and is lower than that previously reported for other MF-IOLs $[6,10,30]$, including trifocal IOLs [31]. The mean value of the disturbance index $(0.08 \pm 0.12)$ provided by the Halo software was low, revealing a low influence of haloes on night vision with this IOL. The value observed in our sample is lower than the corresponding result reported by Carballo-Alvarez in patients with MF-IOLs. In this paper, they reported a mean disturbance index of $0.29 \pm 0.21$ with the FineVision trifocal IOL (Physiol, Belgium) [32]. Hence, our data suggest that the EDOF-IOL of the present study induces less haloes than the trifocal diffractive IOL. However, caution is needed when comparing data from different studies due to differences in the setting of the Halo software.

Finally, it should be noted that a large percentage of patients $(31.6 \%)$ received this EDOF-IOL in one eye only and none of them complained about visual disturbances. Most of these patients had already received a monofocal IOL in the fellow eye. Hence, the Mini Well EDOF-IOL can be considered a safe and effective option also for these patients.

Further investigation is warranted. First, it could be interesting to correlate the functional postoperative results to the preoperative biometric parameters, in order to identify those patients who may have the best results and those who may not be the best candidate for the implantation of such IOL. For example, we could not assess what influence the pupil diameter might have on the visual properties of the EDOF-IOL [12]. Second, it could be interesting to directly compare this new IOL with other MF and EDOF-IOLs in randomized controlled clinical trials.

In conclusion, the Mini Well EDOF-IOL performed well for far distances with no CS loss. Moreover, it provided excellent intermediate vision, better than standard MFIOLs, and good results at near distances, with a low rate of visual disturbances. It can thus be considered a good choice for patients undergoing lens surgery and looking for spectacle independence. 


\section{Summary}

\section{What was known before}

- Traditional MF-IOLs act as bifocal lenses, as they form two primary focal points for distance and near vision, with poor visual function at an intermediate distance.

\section{What this study adds}

- A new extended dept-of-focus multifocal intraocular lens provides a continuum of foci with good vision at intermediate distance.

Acknowledgements The contribution of IRCCS - Fondazione Bietti was supported by Fondazione Roma and the Italian Ministry of Health.

\section{Compliance with ethical standards}

Conflict of interest GS and RB are consultants of SIFI.

\section{References}

1. Savini G, Hoffer KJ, Lombardo M, Serrao S, SchianoLomoriello D, Ducoli P. Influence of the effective lens position, as predicted by axial length and keratometry, on the near add power of multifocal intraocular lenses. J Cataract Refract Surg. 2016;42:44-49.

2. Alfonso JF, Fernández-VegaL, Baamonde MB, Montés-Micó R. Prospective visual evaluation of apodized diffractive intraocular lenses. J Cataract Refract Surg. 2007;33:1235-43.

3. Kohnen T, Nuijts R, Levy P, Haefliger E, Alfonso JF. Visual function after bilater implantation of apodized diffractive aspheric multifocal intraocular lenses with a $+3.0 \mathrm{D}$ addition. J Cataract Refract Surg. 2009;35:2062-9.

4. Maxwell WA, Cionni RJ, Lehmann RP, Modi SS. Functional outcomes after bilateral implantation of apodized diffractive aspheric acrylic intraocular lenses with a +3.0 or +4.0 diopter addition power. J Cataract Refract Surg. 2009;35:2054-61.

5. Pedrotti E, Mastropasqua R, Passilongo M, Parisi G, Marchesoni I, Marchini G. Comparison of two multifocal intraocular lens designs that differ only in near add. J Refract Surg. 2014;30:754-60.

6. Hayashi K, Ogawa S, Manabe S, Hirata A. Visual outcomes in eyes with a distance-dominant diffractive multifocal intraocular lens with low near addition power. Br J Ophthalmol. 2015;99:1466-70.

7. Kretz FTA, Gerl M, Gerl R, Müller M, Auffarth GU, ZKB00 Study Group Clinical evaluation of a new pupil independent diffractive multifocal intraocular lens with a $+2.75 \mathrm{D}$ near addition: a European multicentre study. Br J Ophthalmol. 2015;99:1655-9.

8. Vega F, Alba-Bueno F, Millán MS, Varón C, Gil MA, Buil JA. Halo and through-focus performance of four diffractive multifocal intraocular lenses. Invest Ophthalmol Vis Sci. 2015;56:3967-75.

9. Mojzis P, Kukuckova L, Majerova K, Liehneova K, Piñero DP. Comparative analysis of the visual performance after cataract surgery with implantation of a bifocal or trifocal diffractive IOL. J Refract Surg. 2014;30:666-72.
10. Jonker SMR, Bauer NJC, Makhotkina NY, Berendshot TTJM, van den Biggelaar FJHM, Nuijts RMMA. Comparison of a trifocal intraocular lens with a +3.0 D bifocal IOL: results of a prospective randomized clinical trial. J Cataract Refract Surg. 2015;41:1631-40.

11. Esteve-Taboada JJ, Domínguez-Vicent A, Del Águila-Carrasco AJ, Ferrer-Blasco T, Montés-Micó R. Effect of large apertures on the optical quality of three multifocal lenses. J Refract Surg. 2015;31:666-72.

12. Domínguez-Vicent A, Esteve-Taboada JJ, Del ÁguilaCarrasco AJ, Monsalvez-Romin D, Montés-Micó R. In vitro optical quality comparison of 2 trifocal intraocular lenses and 1 progressive multifocal intraocular lens. J Cataract Refract Surg. 2016;42:138-47.

13. Domínguez-Vicent A, Esteve-Taboada JJ, Del Águila-Carrasco AJ, Ferrer-Blasco T, Montés-Micó R. In vitro optical quality comparison between the Mini Well Ready progressive multifocal and the Tecnis Symfony. Graefes Arch Clin Exp Ophthalmol. 2016;254:1387-97.

14. Bellucci R, Curatolo MC. A new extended depth of focus intraocular lens based on spherical aberration. J Refract Surg. 2017;33:389-94.

15. Savini G, Schiano-Lomoriello D, Balducci N, Barboni P. Visual performance of a new extended depth-of-focus intraocular lens compared to a distance-dominant diffractive multifocal intraocular lens. J Refract Surg. 2018;34:228-35.

16. Camps VJ, Tolosa A, Pinero DP, de Fez D, Caballero MT, Miret JJ. In vitro aberrometric assessment of a multifocal intraocular lens and two extended depth of focus IOLs. J Ophthalmol. 2017;2017:7095734.

17. Calossi A, Boccardo L, Fossetti A, Radner W. Design of short Italian sentences to assess near vision performance. J Optom. 2014;7:203-9.

18. Radner W, Radner S, Raunig V, Diendorfer G. Reading performance of monofocal pseudophakic patients with and without glasses under normal and lim light conditions. J Cataract Refract Surg. 2014;40:369-75.

19. Maaijwee K, Mulder P, Radner W, Van Meurs JC. Reliability Testing of the dutch version of the Radner reading. Optom Vis Sci. 2008;85:353-8.

20. Pepose JS, Qazi MA, Chu R, Stahl J. A prospective randomized clinical evaluation of 3 presbyopia-correcting intraocular lenses after cataract extraction. Am J Ophthalmol. 2014;158: 436-46.

21. Malandrini A, Martone G, Menabuoni L, Catanese AM, Tosi GM, Balestrazzi A, et al. Bifocal refractive corneal inlay implantation to improve near vision in emmetropic presbyopic patients. J Cataract Refract Surg. 2015;41:1962-72.

22. Pomerance GN, Evans DW. Test-retest reliability of the CSV$1000 \mathrm{E}$ contrast test and its relationship to glaucoma therapy. Invest Ophthalmol Vis Sci. 1994;35:3357-61.

23. Gutiérrez R, Jiménez JR, Villa C, Valverde JA, Anera RG. Simple device for quantifying the influence of halos after lasik surgery. J Biomed Opt. 2003;8:663-7.

24. Castro JJ, Jiménez JR, Ortiz C, Alarcón A, Anera RG. New testing software for quantifying discrimination capacity in subjects with ocular pathologies. J Biomed Opt. 2011;16:015001.

25. Villa C, Jiménez JR, Anera RG, Gutiérrez R, Hita E. Visual performance after LASIK for a Q-optimized and a standard ablation algorithm. Appl Opt. 2009;48:5741-5477.

26. McAlinden C, Pesudovs K, Moore JE. The development on an instrument to measure quality of vision: the Quality of Vision (QoV) questionnaire. Invest Ophthalmol Vis Sci. 2010;51: 5537-45.

27. Whittaker SG, Lovie-Kitchin J. Visual requirements for reading. Optom Vis Sci. 1993;70:54-65. 
28. Kamiya K, Hayashi K, Shimizu K, Negishi K, Sato M, BissenMiyajima H. Survey Working Group of the Japanese Society of Cataract and Refractive Surgery. Multifocal intraocular lens explantation: a case series of 50 eyes. Am J Ophthalmol. 2014;158:215-20.

29. Muñoz G, Albarrán-Diego C, Ferrer-Blasco T, Sakla HF, GarcÍaLázaro S. Visual function after bilateral implantation of a new zonal refractive aspheric multifocal intraocular lens. J Cataract Refract Surg. 2011;37:2043-52.

30. Hayashi K, Manabe S, Hayashi H. Visual acuity from far to near and contrast sensitivity in eyes with a diffractive multifocal intraocular lens with a low addition power. J Cataract Refract Surg. 2009;35:2070-1076.

31. Mendicute J, Kapp A, Lévy P, Krommes G, Arias-Puente A, Tomalla M, et al. Evaluation of visual outcomes and patient satisfaction after implantation of a diffractive trifocal intraocular lens. J Cataract Refract Surg. 2016;42:203-10.

32. Carballo-Arvalez J, Vasquez-Molini JM, Sanz-Fernandez J, Garcia-Bella J, Polo V, Garcia-Feijoo J, et al. Visual outcomes after bilateral trifocal diffractive intraocular lens implantation. BMC Ophthalmol. 2015;15:26. 